J. Clin. Chem. Clin. Biochem.

Vol. 18, 1980, pp. 123-127

\title{
Eine schnelle und einfacine nephelometrische Bestimmungsmethode für Protein im Liquor cerebrospinalis
}

Von H. Reiber

Neurochemisches Labor der Neurologischen Klinik und Poliklinik (Direktor: Prof. Dr. H. Bauer), Universität Göttingen

(Eingegangen am 6. Juni/22. September 1979)

Zusammenfassung: Für die nephelometrische Bestimmung der Gesamtproteinkonzentration im Liquor wurden folgende Bedingungen gewählt: $\mathrm{Zu} 550 \mu \mathrm{l}$ einer $2,4 \mathrm{~mol} / 1$ Trichloressigsäurelösung wurden $50 \mu \mathrm{l}$ einer Proteinlösung (2-50 $\mu$ g Protein) zugegeben. Das Streulichtsignal erreicht je nach Proteinkonzentration nach 1-4 Minuten eine maximale Intensität und nimmt dann wieder ab. Diese im Maximum gemessene Streulichtintensität ist linear proportional der Proteinmenge im Testansatz. Eine Trichloressigsäurekonzentration von $2,2 \mathrm{~mol} / 1 \mathrm{im}$ Testansatz stellt ein Optimum sowohl für Streulichtintensität und Reaktionsgeschwindigkeit als auch für die Albumin/GlobulinSpezifität dar.

Die nephelometrische Messung der Proteinfällungskinetik (Vorwärtsstreuung $70^{\circ}, \lambda=400-550 \mathrm{~nm}$, in der Küvette gerührte Reaktionsmischung) ergab eine sehr gute Reproduzierbarkeit: Der Variationskoeffizient für die Bestimmung in Kontrollseren war $1 \%$ und die Von-Tag-zu-Tag-Präzision < 4\%. Lipämische oder hämolytische Proben stören den Test nicht. Der Vergleich mit der Methode nach Lowry für $\mathrm{n}=139$ Liquores ergab eine Regressionsgerade $y=-1,3+0,96 \times(\mathrm{mg} / 1)$ mit einem Korrelationskoeffizienten von $\mathrm{r}=0,944$. Für beide Methoden ist also derselbe Normalbereich der Liquor-Gesamtproteinkonzentration gültig. Die Empfindlichkeit der Methode erlaubt es, aus 20-50 $\mu 1$ Liquor die Gesamtproteinkonzentration direkt, einfach und schnell zu bestimmen. Diese Methode könnte damit die Methode der Wahl für die Gesamtprotein-Bestimmung im Liquor werden.

\section{$A$ rapid and simple nephelometric assay for total protein in cerebrospinal fluid}

Summary: The precipitation of protein from CSF by trichloroacetic acid was optimized by the following procedure. $550 \mu \mathrm{l}$ of a $2.4 \mathrm{~mol} / 1$ trichloroacetic acid solution were continuously stirred in a cuvette, during the addition of $50 \mu \mathrm{l}$ of the sample containing 2-50 $\mu \mathrm{g}$ protein.

The scattered light is measured in a Beckman-Nephelometer $\left(70^{\circ}\right.$ forward scattering at $\left.\lambda=400-550 \mathrm{~nm}\right)$ and recorded.

Within 1-4 minutes scattering reached a maximal intensity followed by a decrease of intensity.

The maximal intensity of the scattered light was found to be linearly proportional to the amount of protein present.

A trichloroacetic acid concentration of $2.2 \mathrm{~mol} / 1$ in the assay was found to be optimal. with respect to a high intensity of scattered light, a fast reaction rate and a comparable specificity for albumins and globulins in the assay.

The coefficient of variation for the determination of control sera was $1 \%$ and the day-to-day precision was $<4 \%$. Lipemic or hemolytic probes did not influence the accuracy.

For $n=139$ specimens of cerebrospinal fluid, the nephelometric method and the common method of Lowry were compared. The regression line was found to be $y=-1.3+0.96 \times(\mathrm{mg} / \mathrm{l})$ with a correlation coefficient of $r=0.944$. This means that for both methods the same reference range is valid.

The sensitivity of the method allows the determination of total protein in 20-50 $\mu \mathrm{l}$ of cerebrospinal fluid. This easy to handle and very rapid method could become the method of choice for the determination of total protein in cerebrospinal fluid. 


\section{Einführung}

Die Gesamtproteinkonzentration im Liquor ist zu niedrig, um die Probe unkonzentriert direkt mit der Biuret-Reaktion, wie sie für Serum angewandt wird (1), zu analysieren.

Zahlreiche nephelometrische, turbidimetrische und kolorimetrische Methoden (2-6) wurden für die Liquoruntersuchungen beschrieben. Am häufigsten wird die Methode nach Lowry et al (4) verwendet. Diese Methode ist aber empfindlich gegen unspezifische Nebenreaktionen, ist langwierig $(1-2 \mathrm{~h})$ und basiert auf einer nichtlinearen Standardkurve.

Die Fällung der Proteine mit Trichloressigsäure und Messung der Trübung oder des Streulichtes wurde schon seit langem beschrieben (Literatur zitiert in 1.c. $(5,6)$ ). Die Hauptschwierigkeit der nephelometrischen Verfahren $(3,5,6)$ liegt darin, daß es nicht gelang, einen stabilen Streulichtendwert zu erhalten. In der vorgelegten $\mathrm{Me}-$ thode wird nun gerade diesem Verlauf der Streulichtkinetik Rechnung getragen und jeweils im Punkte maximaler Streulichtintensität das Streulichtsignal gemessen. Die Wahl einer geeignet hohen Konzentration des Fällungsreagenz macht diese Methode zusätzlich schnell. Die Empfindlichkeit und die Globulin/Albumin-Spezifität sind durch diese Modifikation ebenfalls wesentlich verbessert worden.

Durch die zunehmende Möglichkeit, immunchemisch einzelne Proteinklassen (Albumin, Immunglobuline) im Liquor sehr schnell zu bestimmen (7), wurde die diagnostische Bedeutung des Gesamtproteingehaltes im Liquor immer mehr auf eine grobe Orientierungshilfe reduziert. Für die Notfalldiagnostik und als Orientierung für die Meßbereichswahl der immunchemischen Proteinbestimmungen im Liquor sollte aber die vorliegende Methode durch ihre Schnelligkeit und Einfachheit von besonderem Interesse sein.

\section{Material und Methoden}

Das Streulichtsignal wurde mit dem Nephelometer der Fa. Beckman (7) gemessen ( $70^{\circ}$ Vorwärtsstreuung, $\lambda=400-550 \mathrm{~nm}$ ) und mit angeschlossenem Schreiber registriert.

Trichloressigsäure, p.A., von Merck; Kontrollseren von BehringWerke AG (Fluinorm-N, OTVB. und Protein-Standard-Lösung, OTRH), von Merz und Dade AG (Monitrol I-E und Monitrol II-E), von Hyland Travenol GmbH (Q-Pak I (N), Q-Pak II (P) und Q-Pak control for spinal fluid analysis). Human-Albumin und Immunglobulin (Rind, reinst) waren von Behring Werke AG, $\gamma$-Globulin aus Humanserum $(\approx 97 \%$ rein) und $\beta$-Globulin aus Rinderserum ( $>95 \%$ rein) von Serva.

\section{Methode}

Zu $550 \mu \mathrm{l}$ einer $2,4 \mathrm{~mol} / 1$ Trichloressigsäurelösung in der Rundküvette mit Rührer wurden $50 \mu$ Probenlösung zugegeben. Liquorproben (bis $900 \mathrm{mg} / \mathrm{l}$ ) werden unverdünnt und Serumproben werden 1:200 mit $\mathrm{H}_{2} \mathrm{O}$ oder $9 \mathrm{~g} / 1 \mathrm{NaCl}$-Lösung verdünnt zugegeben.

Die Zugabe der Probe erfolgt in die ins Gerät eingesetzte Küvette bei laufender Registrierung unter kontinuierlichem Rühren. Als
Meßwert wurde die Differenz aus maximalem Streulichtsignal und Leerwert (vor Zugabe der Probe)' ermittelt.

Die so gemessenen relativen Streulichtwerte wurden mit einem 1:200 verdünnten Kontrollserum (Monitrol I-E) in Dreifachbestimmung kalibriert. Für die normale klinische Routinediagnostik reicht aufgrund der Linearität der Standardkurve und des kleinen Variationskoeffizienten von $1 \%$ eine Einfachbestimmung des Standardwertes aus.

Die Von-Tag-zu-Tag-Präzision für das Kontrollserum (unbehandelter Serumpool, 1:200 verdünnt) war $4 \%$ für $n=38$ Bestimmungen. Alle Messungen wurden bèi Räumtemperatur durchgeführt. Eine Thermostatisierung ist nicht nötig, da der Temperatureinfluß auf dạs Streulichtsignal im Bereich $15-35^{\circ} \mathrm{C}<0,4 \% /{ }^{\circ} \mathrm{C}$ beträgt.

Diese Methode ist in jedem Spektralfluorimeter durchfuhrbar, sofern eine Möglichkeit besteht, die Probe kontinuierlich zu rühren oder schnell genug blasenfrei zu mischen.

Für die Vergleichsmessungen nach der Methode von Lowry et al. (4) wurden jeweils drei Standardwerte verschiedener Konzentration aus Fluinorm-N als Standardlösung bestimmt. Die untersuchten Liquores wurden durch Lumbalpunktion von Patienten mit verschiedenen neurologischen Erkrankungen im Rahmen der Routinediagnostik entnommen. Die Zellen wurden abzentrifugiert ( $5 \mathrm{~min}, 1500 \mathrm{~g}$ ) und der Überstand unkonzèntriert zur Proteinbestimmung eingesetzt.

\section{Ergebnisse}

Aus Abbildung 1 ist ersichtlich, daß die Erhöhung der Trichloressiggääurekonzentration als Fällungsireagenz bei gleichbleibender Proteinkonzentration die Größe und aüch den zeitaḅhängigen Verlauf der Streulichtintensität stark beeinflußt.

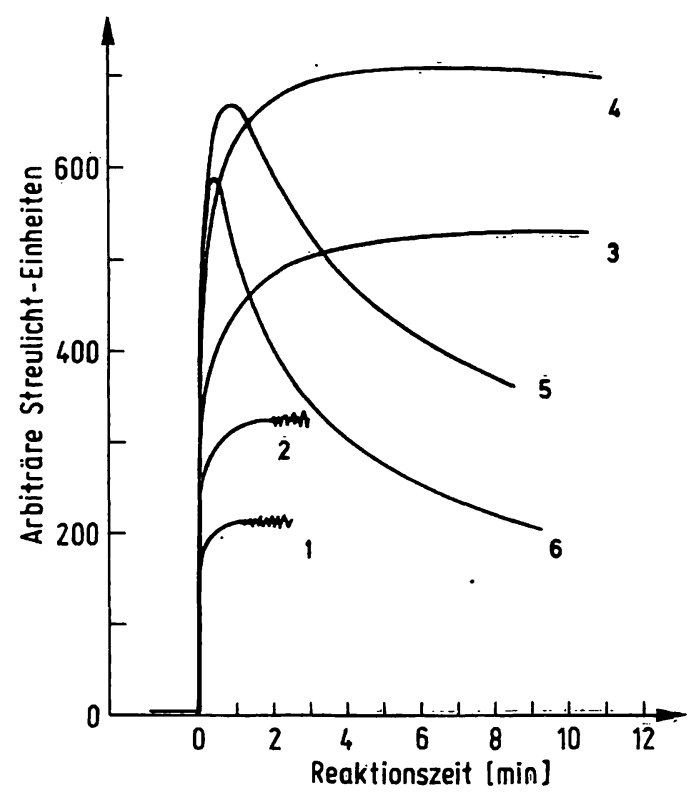

Abb. 1. Einfluß der Trichloressigsäurekonzentration auf die Proteịnfällung.

Zu $550 \mu \mathrm{l}$ Trichloressigsäurelösung verschiedener Konzentrationen wurden $50 \mu \mathrm{l}$ einer Serumprobe (verdünnt mit $9 \mathrm{~g} / 1 \mathrm{NaCl}$-Lösung auf $700 \mathrm{mg} / \mathrm{l}$ ) zugegeben. Die Trichloressigsäurekonzentrationen im Test (in mol/1) sind für die Kurven $1 \ldots 0,6 ; 2 \ldots 0,78 ; 3 \ldots 1,02 ; 4 \ldots 1,63$; $5 \ldots 2,0 ; 6 \ldots 2,2$. Das in den Kurven 1 und 2 sichtbare starke Rauschen des Streulichtsignals kommt durch das Ausflocken dêr Proteinsuspension. Das Streulichtsignal wurde mit dem Nephelometer der Fa. Beckman $\left(70^{\circ}\right.$ Vorwärtsstreuung, $\lambda=400-550 \mathrm{~nm}$ ) unter kontinuierlichem Rühren der Reaktionsmischung mit dem Schreiber registriert. 
Das hat verschiedene Gründe: Bei sehr niedrigen Trichloressigsäurekonzentrationen $(<0,4 \mathrm{~mol} / \mathrm{l})$ ist die Proteinfällung nicht quantitativ. Das wurde daraus ersichtlich, daß nach Abzentrifugieren der 1. Fällung und anschlieBender Erhöhung der Trichloressigsäurekonzentration weiteres Protein ausgefällt wurde. Durch die Erhöhung der Temperatur kann ebenfalls, gerade bei niedrigen Trichloressigsäurekonzentrationen, eine vollständigere Proteinausfällung erreicht werden.

Bei mittleren Trichloressigsäurekonzentrationen (0,6$0,78 \mathrm{~mol} / 1$, Kurven 1 und 2 in Abb. 1) ist zwar die Ausfällung quantitativ, aber die Proteinpartikel agglomerieren und bilden eine flockige Suspension, die das in Abbildung 1, Kurve 1 und 2 erkennbare starke Rauschen des Streulichtsignals bewirken.

Bei höheren Konzentrationen der Trichloressigsäure (Kurven 3 und 4 in Abb. 1) nimmt die Streulichtintensität weiter zu, ein Ausflocken wurde im Meßzeitraum nicht beobachtet. Bei noch höheren Konzentrationen. (Kurven 5 und 6 Abb. 1) durchläuft das Streulichtsignal ein Intensitätsmaximum. Obwohl das Streulichtsignal bereits nach wenigen Minuten wieder abnimmt, nimmt die Trübung des Reaktionsgemisches (Messung der optischen Dichte) weiter zu und erreicht ein Maximum erst nach mehr als 30 Minuten.

Das Maximum der Streulicht-Intensitätskurven (4-6 in Abb. 1) wird mit wachsender Trichloressigsäurekonzentration zunehmend früher erreicht. Dabei nimmt allerdings das maximale Streulichtsignal wieder ab. Bei Konzentrationen $>2,75 \mathrm{~mol} / 1$ wird dieses Signal sogar null (keine Proteinfällung).

Als optimaler Kompromiß für eine möglichst schnelle Reaktion mit möglichst großem Streulichtsignal wurde entsprechend Kurve 6 in Abbildung 1 eine 2,4 mol/1 Trichloressigsäure-Reagenzlösung $(2,2 \mathrm{~mol} / 1$ bezogen auf den Testansatz) gefunden.

In Abbildung 2 ist nun zu erkennen, daß mit wachsender Proteinkonzentration im Test, bei konstanter Trichloressigsäurekonzentration, die Streulichtintensität im Intensitätsmaximum zunimmt und außerdem der Zeitpunkt, an dem das Maximum erreicht wird, verschoben wird.

Die jeweils maximale Streulichtintensität der Kurven in Abbildung 2 wurde nun in Abbildung 3 als Funktion der Proteinmenge dargestellt. Dieser lineare Zusammenhang über den gesamten Meßbereich und die sehr gute Reproduzierbarkeit sind eine wichtige Voraussetżung für die bequeme Handhabung dieser Methode.

Für diese Reproduzierbarkeit der Meßwerte (VK = $1 \%$ ) ist allerdings notwendig, daß die Probe kontinuierlich gerührurt wird. Der Beitrag der unspežifísschen Eigentrübung der Serum- oder Liquorproben zum Streulichtsignal kann oberhalb einer Proteinkonzentration von $5 \mu \mathrm{g} /$ Test vernachlässigt werden. Dies ist für den klinischen Bedarf immer der Fall. Für eine starke Eigentrübung bei sehr

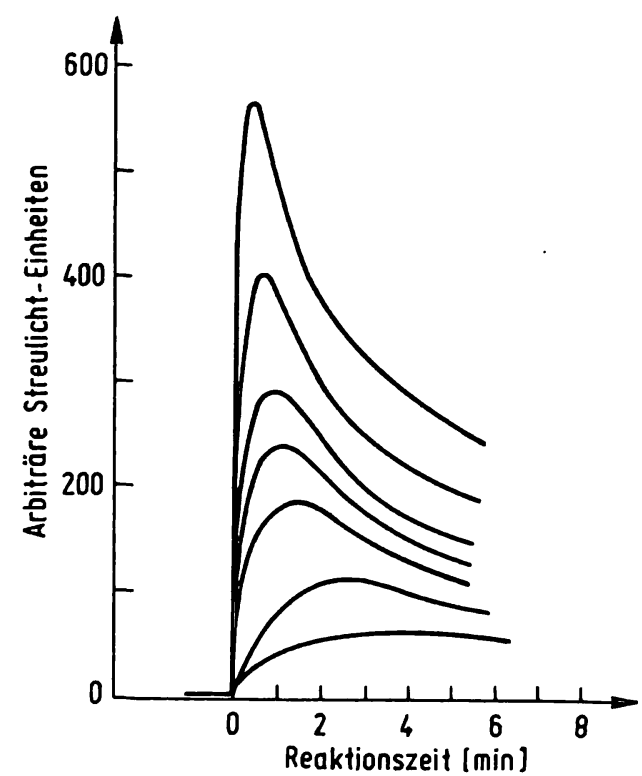

Abb. 2. Einfluß der Proteinmenge auf die Kinetik der Proteinfällung.

Zu $550 \mu \mathrm{l}$ 2,4 mol/1 Trichloressigsäurelösung wurden $50 \mu \mathrm{l}$ entsprechend verdünntes Serum (Serumpool) zugegeben, um die folgenden Gesamtproteinmengen im Test zu haben: von unten nach oben 3,5 / 7,0 / 10,5 / $14 / 17,5 / 23,5 / 35 \mu \mathrm{g}$. Meßmethode wie in Legende von Abbildung 1 beschrieben.

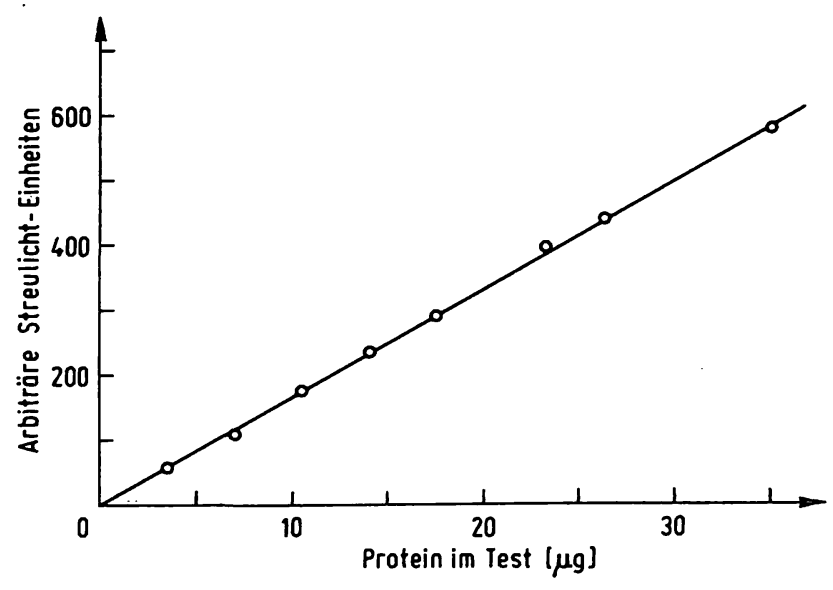

Abb. 3. Maximale Streulichtintensität als Funktion der Proteinmenge im Test.

Für die in Abbildung 2 gezeigten Kurven wurde die Streulichtintensität im Maximum gemessen, davon der Streulichtleerwert (vor Zugabe der Probe) abgezogen und als Funktion der jeweiligen Gesamtproteinmenge im Test aufgetragen. Die Kreise charakterisieren den Mittelwert einschließlich Standardabweichung aus drei unabhängigen Meßreihen. Reaktionsansätze und Meßmethode siehe Legende von Abbildung 1.

kleinem Streulichtsignal könnte aber korrigiert werden, wenn zusätzlich ein Probenleerwert in $550 \mu \mathrm{H} \mathrm{H}_{2} \mathrm{O}$ (statt Trichloressigsäure) gemessen und vom Meßwert abgezogen wird. Bei Zumischung von Albumin zu Serum oder Liquor werden $100 \%$ wiedergefunden. Stark hämolytische Proben stören ebenfalls nicht. 98 \% einer Protein- 
mischung (Monitrol I) wurden in einer Probe mit hämolysiertem Vollblut wiedergefunden. Da das Streulicht gemessen wird (also nicht die Trübung), und der innere Filtereffekt aufgrund der starken Verdünnung sehr klein wird, ist verständlich, daß diese Effekte vernachlässigbar werden.

Die Trichloressigsäurekonzentration von $2,2 \mathrm{~mol} / 1 \mathrm{im}$ Testansatz stellt aus verschiedenen Gründen ein Optimum dar. Neben der beschriebenen Beschleunigung der Reaktionsgeschwindigkeit und der Vergrößerung des Streulichtsignals werden bei dieser Konzentration des Reagenz $(2,2 \mathrm{~mol} / \mathrm{l})$ auch vergleichbare Streulichtintensitäten für Albumine und Globuline erreicht: Für gleiche Mengen Protein verhalten sich die relativen Streulichtintensitäten von Albumin : Immunglobulin : $\boldsymbol{\gamma}$-Globulin : $\beta$-Globulin $=1: 1: 0,9: 0,6$. Bei einer Reagenzkonzentration von $1,65 \mathrm{~mol} / 1 \mathrm{im}$ Testansatz werden dagegen die Albumine bevorzugt nachgewiesen: Albumin : Immunglobulin : $\gamma$-Globulin : $\beta$-Globulin $=1: 0,4: 0,8: 0,4$. Bei hohen Reagenzkonzentrationen, z. B. bei $2,75 \mathrm{~mol} / 1$, werden die Globuline bevorzugt nachgewiesen, entsprechend 1:6:6:4. Der Vergleich verschiedener Kontrollseren ergab ebenfalls für eine Trichloressigsäurekonzentration von 2,2 $\mathrm{mol} / 1$ die beste Vergleichbarkeit: Bezogen auf Monitrol I-E als Standardserum ergaben sich folgende Abweichungen von den Sollwerten: Monitrol II-E $(-4,5 \%)$, Fluinorm-N $(+1,0 \%)$, Q-Pak I $(-1,5 \%)$, Q-Pak II $(-1,0 \%)$, Proteinstandard-Lösung $(+7,3 \%)$, Q-Pak control for cerebrospinal fluid analysis $(+4,2 \%)$ und ein unbehandelter Serumpool $(+4,4 \%)$.

Für die Liquores von $n=139$ Patienten verschiedener neurologischer Erkrankungen wurde der Gesamtproteingehalt mit der nephelometrischen Methode und nach der herkömmlichen Bestimmungsmethode nach Lowry et al (4) bestimmt. Der Vergleich ist in Abbildung 4 dargestellt. Daraus ist ersichtlich, daß für beide Methoden dieselben Normalbereiche des Gesamtproteingehaltes im Liquor gültig sind. Die Von-Tag-zu-Tag-Präzision der nephelometrischen Methode entspricht mit 3,8\% der Präzision der Lowry-Methode (3,9\%).

\section{Diskussion}

Die beschriebene Charakteristik der Proteinfällung in Anwesenheit hoher Trichloressigsäurekonzentrationen, für die eine Abnahme der Streulichtintensität nach Erreichen eines Maximums typisch ist (Kurven 4-6 in Abb. 1), wird nur durch Streulichtmessung sichtbar. Die Mesşung der Trübungszunahme (Turbidimetrie) zeigt dagegen den für eine Sättigungskurve typischen Verlauf. Eine detaillierte Interpretation dieser Kurvenverläufe ist

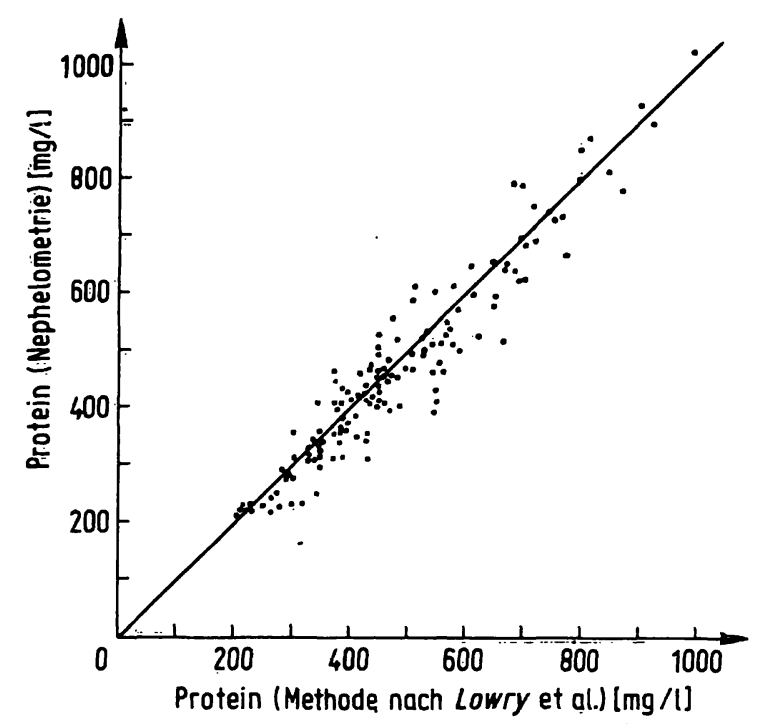

Abb. 4. Methodenvergleich für die Gesamtproteinkonzentrạtion im Liquor.

Für n $=139$ unausgewählte Liquores wurde der Gesamtproteingehalt mit der in dieser Arbeit beschriebenen nephelometrischen Methode und der Methode nach Lowry et al (4) bestimmt. Die eingezeichnete Gerade ist die theoretische Gerade $y=-1,3+0,96 \times(\mathrm{mg} / \mathrm{l}) \mathrm{mit}$ einem Korrelationskoeffizienten von $r=0,944$.

nicht Gegenstand dieser Untersuchung. Es ist jedoch aus theoretischen Gründen klar, daß die beobachteten Veränderungen der Streulichtintensität eine Funktion der sich verändernden Partikelgrößen und Partikelzahl der ausgefällten Proteine sind (8).

Der Vorteil der nephelometrischen Methode besteht weiterhin auch darin, daß der Einfluß von trüben, xanthochromen oder hämolytischen Proben auf das Ergebnis vernachlässigbar wird.

Der Meßbereich dieser Methode reicht von $2-50 \mu \mathrm{g}$ Protein/Test. Die obere Grenze wird durch die apparativen Bedingungen (maximales Streulichtsignal entspricht 1000 relativen Streulichteinheiten) festgelegt. Bei einem Probenvolumen von $50 \mu \mathrm{l}$ können Liquores mit einer Gesamtproteinkonzentration bis $900 \mathrm{mg} / \mathrm{l}$ unverdünnt analysiert werden.

Durch die Schnelligkeit, Einfachheit in der Durchführung und die Reproduzierbarkeit der Ergebnisse ist diese Methode für uns die Methode der Wahl für die Bestimmung der Gesamtproteinkonzentration im Liquor gewordèn.

\section{Danksagung}

Ich danke Herrn P. Lange für seine kreative technische Assistenz. 


\section{Literatur}

1. Doumas, B. T. (1975), Clin. Chem. 21, 1159.

2. Richterich, R. \& Colombo, J. P. (1978), Klinische Chemie, Karger-Verlag, 4. Aufl. S. 527.

3. Bauer, H. \& Angelstein, I. (1952), Klin. Wochenschr. 30, 277-279.

4. Lowry, O. H., Rosebrough, N. J., Farr, A. L. \& Randall, R. J. (1951), J. Biol. Chem. 193, 265-275.
5. Bauer, H. (1957), Dtsch. Z. Nervenhkd. 176, 126-142.

6. Henry, R. J., Clinical Chemistry Principles and Techniques, Harper and Row, 1967, S. 188-189.

7. Sternberg, J. C. (1977), Clin. Chem. 23, 1456-1464.

8. Van Holde, K. E. (1971), Physical Biochemistry, Foundations of Modern Biochemistry Series, Prentice-Hall, Inc., Englewood Cliffs, N.J.S. 180-201.

Dr. H. Reiber

Neurochemisches Labor der Neurolog. Klinik Robert-Koch-Str. 40

D-3400 Göttingen 
. 